\title{
All-Cause, Stroke-, and Bleed-Specific Healthcare Costs: Comparison among Patients with Non-Valvular Atrial Fibrillation (NVAF) Newly Treated with Dabigatran or Warfarin
}

\author{
Adrienne M. Gilligan ${ }^{1}$ - Pranav Gandhi ${ }^{2} \cdot$ Xue Song $^{1} \cdot$ Cheng Wang $^{2}$ • \\ Caroline Henriques $^{1} \cdot$ Stephen Sander $^{2} \cdot$ David M. Smith $^{1}$
}

Published online: 9 August 2017

(C) The Author(s) 2017. This article is an open access publication

\begin{abstract}
Objective Our objective was to compare all-cause and stroke- and bleed-specific healthcare costs among patients with non-valvular atrial fibrillation (NVAF) treated with dabigatran or warfarin.

Methods Administrative claims data from the MarketS$\mathrm{can}^{\circledR}$ Databases for 2009-2014 were used. Patients with NVAF newly treated with dabigatran were matched 1:1 to those treated with warfarin. All-cause and stroke- and bleed-specific costs per patient per month (PPPM) (\$US, year 2015 values) up to a 12-month follow-up period were analyzed. Stroke- or bleed-specific costs were defined as hospitalizations with stroke or bleed as the primary discharge diagnosis and outpatient claims with stroke or bleed diagnosis in any position. Differences in costs between dabigatran and warfarin users were assessed using descriptive and multivariate analyses.
\end{abstract}

Data from this study were presented as abstract \#I08 at the Academy of Managed Care Pharmacy Nexus 2016 congress, October 3-6, 2016, National Harbor, MD, USA, and as abstract \#532 at the 58th Annual Meeting of the American Society of Hematology 2016, December 3-6, 2016, San Diego, CA, USA.

Electronic supplementary material The online version of this article (doi:10.1007/s40256-017-0244-1) contains supplementary material, which is available to authorized users.

\section{Adrienne M. Gilligan}

agilliga@us.ibm.com

1 Truven Health Analytics, an IBM Company, 150 Cambridge Park Dr, Cambridge, MA 02140, USA

2 Boehringer-Ingelheim Pharmaceuticals, Inc., Ridgefield, CT, USA
Results A total of 18,980 dabigatran-treated patients were matched to corresponding warfarin-treated patients. Adjusted all-cause total healthcare, inpatient, and outpatient costs were significantly lower for the dabigatran cohort (\$US3053 vs. 3433; \$US904 vs. 1194; \$US1594 vs. 1894, respectively; all $p<0.001$ ), but mean pharmacy costs were significantly higher (\$US556 vs. 345, $p<0.001$ ). Stroke-specific total healthcare and outpatient costs were significantly lower for the dabigatran than for the warfarin cohort (\$US30.37 vs. 40.99 and \$US7.36 vs. 12.20, respectively; $p<0.05$ for both values). Similarly, bleed-specific total healthcare and inpatient costs were significantly lower for the dabigatran than for the warfarin cohort (\$US50.00 vs. 73.49 and \$US27.75 vs. 48.66, respectively; $p<0.01$ for both values).

Conclusion Patients receiving dabigatran had significantly lower total all-cause, inpatient, and outpatient costs but higher pharmacy costs than those receiving warfarin. In addition, stroke-specific total and outpatient costs and bleed-specific total and inpatient costs were significantly lower in dabigatran users compared with warfarin users. 


\section{Key Points}

This study examined all-cause and stroke- and bleedspecific healthcare costs among patients with newly diagnosed non-valvular atrial fibrillation newly treated with dabigatran or warfarin.

Compared with previous studies that compared costs among patients receiving dabigatran or warfarin, the present study contains a larger sample size (almost 19,000 per treatment cohort) and was adequately powered and designed to detect potential differences among stroke-specific and bleed-specific outcomes.

Dabigatran users had significantly lower all-cause, inpatient, and outpatient but higher pharmacy costs compared with warfarin users.

Dabigatran users had significantly lower strokespecific total and outpatient and bleed-specific total and inpatient costs compared with warfarin users.

\section{Introduction}

Atrial fibrillation (AF) is the most common clinical dysrhythmia and affects approximately 6 million people in the United States (US) [1, 2]. Estimates suggest that over 150,000 new cases of AF are reported each year [3]. Nonvalvular $\mathrm{AF}$ (NVAF) is the most prevalent type of $\mathrm{AF}$ and accounts for $95 \%$ of diagnosed cases of AF in the USA [4]. NVAF is associated with a five times higher risk of ischemic stroke and accounts for up to $25 \%$ of strokes occurring after the age of 80 years $[4,5]$. Because the risk of stroke increases substantially in patients with NVAF, stroke prevention is crucial in reducing mortality and disability in these patients [6].

NVAF imposes a significant resource and cost burden on the US healthcare system. Approximately 750,000 hospitalizations and 5 million office visits each year are due to NVAF, leading to an economic burden of over \$US6 billion (year 2015 values) $[1,7]$. The mean incremental costs for patients with NVAF and stroke relative to those without stroke were estimated to be more than \$US26,000 annually [8]. Furthermore, compared with patients without a stroke or bleeding event, the average incremental costs in the first year after a stroke or bleeding event in Medicare beneficiaries were found to be $\$$ US32,900 for ischemic stroke, \$US23,414 for major bleeding, and \$US47,640 for intracranial hemorrhage with costs [9].

The treatment goals of NVAF management are to prevent stroke, maintain sinus rhythm, and provide symptomatic relief $[10,11]$. The American College of Chest Physicians antithrombotic guidelines recommend oral anticoagulation rather than no therapy, aspirin, or combination therapy of aspirin with clopidogrel for patients with an intermediate or high risk of stroke as defined by the $\mathrm{CHADS}_{2}$ (Congestive heart failure, Hypertension, Age, Diabetes, prior Stroke) score [12]. Dabigatran, rivaroxaban, apixaban, and edoxaban are novel oral anticoagulants (NOACs) that were approved in October 2010, July 2011, October 2012, and January 2015, respectively, for the prevention of stroke in patients with NVAF. Before these NOACs were approved, warfarin was the primary anticoagulant used [13] and, when well-managed by the healthcare provider, has been shown to be superior to usual medical care compared with less wellmanaged warfarin [14]. In addition to being superior to warfarin for ischemic stroke and less likely to cause hemorrhagic strokes [15-18], these NOACs have other advantages, including fewer interactions with food and other drugs, rapid onset, and freedom from the need for periodic blood test monitoring [19]. While pharmacy costs are higher for NOACs, these higher costs are offset by lower utilization and other healthcare costs (i.e., inpatient and outpatient visits and readmissions) [20, 21].

Data from the RE-LY (Randomized Evaluation of LongTerm Anticoagulant Therapy) clinical trial suggested that dabigatran $150 \mathrm{mg}$ twice daily reduced the risk of stroke, systemic embolism, and intracranial hemorrhage compared with warfarin but increased the risk of major gastrointestinal hemorrhage $[15,16,22,23]$. Similar findings have been demonstrated in several observational studies $[18,24,25]$, whereas other studies have found dabigatran to be comparable in preventing ischemic stroke relative to warfarin [26, 27]. Although real-world evidence assessing the all-cause cost of dabigatran among patients with NVAF compared with warfarin are available [20,21], real-world data specifically evaluating stroke- and bleed-specific healthcare costs among patients with NVAF treated with dabigatran or warfarin are limited, and these studies were not powered to detect stroke- or bleed-specific costs $[20,21]$. This retrospective analysis sought to compare allcause, stroke-, and bleed-specific costs among patients with NVAF newly treated with dabigatran or warfarin.

\section{Methods}

\subsection{Study Design and Data Source}

This was a retrospective matched-cohort study based on administrative claims data from the Truven Health MarketScan ${ }^{\circledR}$ Commercial Claims and Encounters and Medicare Supplemental and Coordinated Benefits databases 
between 1 July 2009 and 31 December 2014. The two databases include cost, utilization, and outcomes data in both inpatient and outpatient settings for millions of patients insured commercially or who have Medicare supplemental insurance paid by employers. The medical claims are linked to outpatient prescription drug claims and person-level enrollment data through the use of unique enrollee identifiers. All database records were de-identified and fully complied with the Health Insurance Portability and Accountability Act (HIPAA) of 1996. Because this study did not involve the collection, use, or transmittal of individually identifiable data, it was exempt from Institutional Review Board approval.

\subsection{Patient Selection}

Patients were included in the study if they had at least one inpatient or two outpatient claims of AF diagnosis (International Classification of Diseases and Related Health Problems, ninth revision, clinical modification [ICD-9CM] code 427.31) between 1 January 2010 and 31 December 2013 (identification period). The two outpatient claims were required to be at least 30 days apart but within 1 year. To ensure the NVAF was newly diagnosed, all patients were required to have been continuously enrolled for at least 6 months before the first observed AF diagnosis, to have no diagnosis of AF and no evidence of cardiac surgery, pulmonary embolism, valvular heart disease, hyperthyroidism, pregnancy, valve replacement, pericarditis, or myocarditis. Patients were further required to have at least two outpatient pharmacy claims for dabigatran or warfarin on or after the first observed AF diagnosis between 1 January 2011 and 31 December 2013 (index period). The date of the first prescription claim for dabigatran or warfarin was designated as the index date. All patients had to be continuously enrolled for at least 12 months prior to the index date (pre-index period). To ensure patients were newly treated, those with pharmacy claims for oral anticoagulants (OACs) during the 12-month pre-index period were excluded from the study. Patients were followed for up to 12 months until index therapy discontinuation or switching from the index OAC to a different anticoagulant, disenrollment, end of study period (i.e., 31 December 2014), or inpatient death. Patients aged $<18$ years on the index date were excluded.

\subsection{Outcome Measures}

All-cause, stroke-, and bleed-specific healthcare costs were assessed in the up to 12-month follow-up period for both the dabigatran- and the warfarin-treated cohorts. Strokespecific costs were defined as hospitalizations with stroke as the primary discharge diagnosis (hospitalizations with stroke as secondary diagnoses were not included to avoid overestimation) and outpatient claims with stroke diagnosis in any position (because the order of diagnosis is random in outpatient claims; there is no distinction between primary or secondary diagnosis). Bleed-specific costs were defined similarly, based on bleeding diagnosis codes (Table S1 in the Electronic Supplementary Material [ESM]). Selection of stroke- and bleed-specific ICD-9-CM diagnosis codes was consistent with previous studies [22, 23].

To account for the variable length of the follow-up period, all healthcare services costs were reported as mean costs per patient per month (PPPM). The PPPM cost was calculated as the total cost for each patient during the follow-up period and then divided by the number of months of follow-up for the patient. PPPM is a common method used in multiple therapeutic areas in outcomes research [20, 28-33]. Costs were reported among all patients, including those with no costs. All-cause cost measures included total (inpatient, outpatient, and outpatient pharmacy) costs. Outpatient costs consisted of emergency room visit costs, outpatient office visit costs, and other outpatient visit costs. Stroke- and bleed-specific cost measures included total costs (inpatient and outpatient, where outpatient costs included emergency room visit costs, outpatient office visit costs, and other outpatient visit costs). Costs were the total reimbursed amount, including patient deductibles, copayments, and coordination of benefits. All healthcare costs were in \$US and adjusted to year 2015 values using the Medical Care component of the Consumer Price Index.

\subsection{Covariates}

Predictor variables consisted of demographic and clinical characteristics. Patient demographics such as age, sex, primary payer (commercial or Medicare), health plan type, and geographic location (US census division) were measured at the index date.

Clinical characteristics were measured during the 12-month pre-index period, including (1) Deyo-Charlson Comorbidity Index (DCCI) score; (2) proxy measures of overall health status (number of unique medications, inpatient hospitalizations, physician visits, or presence of hip fracture); (3) chronic conditions (i.e., chronic kidney disease [CKD], chronic obstructive pulmonary disease [COPD], cirrhosis/hepatitis, coronary artery disease [CAD], diabetes mellitus, heart failure [HF], hypertension, myocardial infarction [MI], paraplegia/hemiplegia, psychiatric disorders, deep vein thrombosis [DVT]); (4) stroke-specific conditions (ischemic or hemorrhagic); (5); bleed-specific conditions (intracranial, extracranial, or gastrointestinal bleed); and (6) having one or more outpatient prescriptions for selected drug classes (i.e., beta 
blockers, calcium channel blockers, diuretics, other antihypertensive, antihyperlipidemics, steroids, antidiabetics, anti-arrhythmics, ketoconazole, and antiplatelets). In addition, the $\mathrm{CHADS}_{2}$ and HAS-BLED scores were included to account for stroke and bleed risk [27]. $\mathrm{CHADS}_{2}$ was scored on a scale of $0-6$ based on the presence and weighting of the following clinical conditions: congestive heart failure, hypertension, age, diabetes, and stroke [27]. Bleed risk was assessed using the HASBLED and ATRIA scores [34]. HAS-BLED was scored on a scale of 0-9 based on the presence and weighting of the following clinical conditions: hypertension, abnormal renal/liver function, stroke, bleed history or predisposition, labile international normalized ratio (INR), elderly, and drugs/alcohol concomitantly [27]. ATRIA scores ranged from 0 to 10 based on the presence and weighting of the following clinical conditions: anemia, severe renal disease, age, hemorrhagic diagnosis, and hypertension [34]. ICD-9 diagnosis codes and national drug codes (NDCs) were used as proxies for elements of the $\mathrm{CHADS}_{2}$, HAS-BLED, and ATRIA scores. Number of days between the first AF diagnosis and the index date and baseline total all-cause expenditures were also captured and controlled for in the analyses.

\subsection{Statistical Analyses}

All study variables were summarized descriptively for each treatment cohort. Data for categorical variables were summarized as counts and percentages, and those for continuous variables were presented as means and standard deviations. Statistical comparisons were evaluated using Chi-squared tests for categorical variables and Student's $t$ tests for continuous measures. A critical value of $p<0.05$ was specified a priori as the threshold for statistical significance. Sensitivity analyses examined patients with outlier costs (for example, the top 1\%) for the descriptive analyses.

A challenge with retrospective cohort studies in general, and with this study in particular, is the question of comparability of patient groups. Differences in patient characteristics that influence the progression and management of NVAF can confound outcomes such as costs. Thus, propensity score matching using the nearest neighbor with caliper was conducted to match dabigatran and warfarin users in a 1:1 ratio [35]. Propensity scores were calculated by logistic regression analyses using demographic characteristics (e.g., age group, sex, health plan type, and region) and clinical characteristics, including DCCI, number of unique generic drugs prescribed, number of inpatient stays, number of office visits, a diagnosis of fracture, CKD, COPD, cirrhosis/hepatitis, CAD, diabetes, HF, hypertension, MI, paraplegia/hemiplegia, psychiatric disorder, venous thrombosis, stroke-specific conditions (ischemic or hemorrhagic bleed), bleed-specific conditions (intracranial, extracranial, or gastrointestinal bleed), $\mathrm{CHADS}_{2}$ score, HAS-BLED score, total costs at baseline, days from first $\mathrm{AF}$ diagnosis to index date, and prescription for beta blockers, calcium channel blockers, diuretics, hypertensives, antihyperlipidemics, steroids, antidiabetics, anti-arrhythmics, ketoconazole, and antiplatelets. These matching factors were selected based on descriptive analysis results (Table 1; Table S2 in the ESM).

Following propensity score matching, multivariate analyses were conducted on the matched cohorts to control for any remaining imbalances in observed covariates that might affect the outcome estimates. Generalized linear models (GLMs) with Gamma distribution and log-link were used to assess the marginal impact of dabigatran versus warfarin on all-cause healthcare costs (including total, inpatient, total outpatient visits, and outpatient prescription costs). The covariates in the GLMs were matching factors plus an indicator of dabigatran versus warfarin. Multivariate analyses were not performed for the strokespecific and bleed-specific costs because the sample size of patients with stroke-specific and bleed-specific events was small.

\section{Results}

\subsection{Patient Selection}

A total of 63,862 patients with newly diagnosed NVAF were identified between 1 January 2010 and 31 December 2013. Of these, 20,348 (31.9\%) patients were newly treated with dabigatran and 43,514 (68.1\%) were newly treated with warfarin. After propensity score matching, 18,980 matched pairs were included for this study. All results were reported separately for matched dabigatran- and warfarintreated patients. The pre- and post-match characteristics of each cohort for the matching variables are reported in Table 1 and in Table S2 in the ESM.

\subsection{Patient Characteristics}

Table 1 summarizes the pre- and post-matching demographic and clinical characteristics of patients newly diagnosed with NVAF and newly treated with dabigatran and warfarin. After matching, most patient demographic and clinical characteristics were similar. However, patients receiving dabigatran were significantly younger $(67.8 \pm$ standard deviation [SD] 11.9 vs. $68.1 \pm 12.0$, respectively; $p<0.05)$ and were less likely to have Medicare as their primary payer (57.0 vs. $58.3 \%$, respectively; $p<0.05$ ). The majority of patients in each cohort 
Table 1 Demographic and clinical characteristics among patients with non-valvular atrial fibrillation before and after propensity score matching

\begin{tabular}{|c|c|c|c|c|}
\hline \multirow[t]{2}{*}{ Characteristics } & \multicolumn{2}{|l|}{ Pre-matching } & \multicolumn{2}{|l|}{ Post-matching } \\
\hline & $\begin{array}{l}\text { Dabigatran } \\
(n=20,348)\end{array}$ & $\begin{array}{l}\text { Warfarin } \\
(n=43,514)\end{array}$ & $\begin{array}{l}\text { Dabigatran } \\
(n=18,980)\end{array}$ & $\begin{array}{l}\text { Warfarin } \\
(n=18,980)\end{array}$ \\
\hline Age (years) & $67.5 \pm 11.9$ & $71.8 \pm 12.1^{* *}$ & $67.8 \pm 11.9$ & $68.1 \pm 12.0 *$ \\
\hline Male & $12,943(63.6)$ & $25,048(57.6)^{* *}$ & $11,962(63.0)$ & $12,029(63.4)$ \\
\hline \multicolumn{5}{|l|}{ Payer } \\
\hline Commercial & $8979(44.1)$ & $12,780(29.4)^{* *}$ & $8164(43.0)$ & $7919(41.7)^{*}$ \\
\hline Medicare & $11,369(55.9)$ & $30,734(70.6)^{* *}$ & $10,816(57.0)$ & $11,061(58.3)^{*}$ \\
\hline \multicolumn{5}{|l|}{ Geographic region of residence } \\
\hline Northeast & $4276(21.0)$ & $9158(21.1)$ & $4003(21.1)$ & $4070(21.4)$ \\
\hline North central & $5635(27.7)$ & $14,590(33.5)^{* *}$ & $5377(28.3)$ & $5468(28.8)$ \\
\hline South & $7417(36.5)$ & $11,733(27.0)^{* *}$ & $6720(35.4)$ & $6599(34.8)$ \\
\hline West & $2886(14.2)$ & $7806(17.9)^{* *}$ & $2760(14.5)$ & $2717(14.3)$ \\
\hline Unknown & $134(0.7)$ & $227(0.5)^{*}$ & $120(0.6)$ & $126(0.7)$ \\
\hline Died within 12 months of index & $130(0.5)$ & $551(1.3)^{* *}$ & $102(0.5)$ & $134(0.7)^{*}$ \\
\hline $\begin{array}{l}\text { Days between first AF diagnosis to index } \\
\text { date }\end{array}$ & $119.9 \pm 211.6$ & $148.6 \pm 248.6^{* *}$ & $121.2 \pm 213.4$ & $124.5 \pm 220.9$ \\
\hline \multicolumn{5}{|l|}{ Comorbid conditions } \\
\hline Hypertension & $13,831(68.0)$ & $31,559(72.5)^{* *}$ & $12,942(68.2)$ & $12,921(68.1)$ \\
\hline Coronary artery disease & $6195(30.5)$ & $16,331(37.5)^{* *}$ & $5865(30.9)$ & $5960(31.4)$ \\
\hline Diabetes mellitus & $5354(26.3)$ & $13,879(31.9)^{* *}$ & $5059(26.7)$ & $5065(26.7)$ \\
\hline Psychiatric disorders & $3921(19.3)$ & $10,442(24.0)^{* *}$ & 3714 (19.6) & $3687(19.4)$ \\
\hline Heart failure & $3677(18.1)$ & $12,673(29.1)^{* *}$ & 3526 (18.6) & 3538 (18.6) \\
\hline COPD & $3473(17.1)$ & $10,043(23.1)^{* *}$ & $3291(17.3)$ & $3311(17.4)$ \\
\hline Bleed-specific conditions & $1826(9.0)$ & $6291(14.5)^{* *}$ & $1748(9.2)$ & $1769(9.3)$ \\
\hline Chronic kidney disease & $1277(6.3)$ & $6785(15.6)^{* *}$ & $1235(6.5)$ & $1241(6.5)$ \\
\hline Myocardial infarction & $1179(5.8)$ & $4562(10.5)^{* *}$ & $12,942(68.2)$ & $12,921(68.1)$ \\
\hline Stroke-specific conditions & $1181(5.8)$ & $4298(9.9)^{* *}$ & $1140(6.0)$ & $1182(6.2)$ \\
\hline Paraplegia/hemiplegia & 324 (1.6) & $1646(3.8)^{* *}$ & $314(1.7)$ & $303(1.6)$ \\
\hline Hemiplegia & $315(1.6)$ & $1571(3.6)^{* *}$ & $306(1.6)$ & $292(1.5)$ \\
\hline Deep vein thrombosis & $301(1.5)$ & $3640(8.4)^{* *}$ & $295(1.6)$ & $301(1.6 \%$ \\
\hline Cirrhosis/hepatitis & $274(1.4)$ & $813(1.9)^{* *}$ & $260(1.4)$ & $253(1.3)$ \\
\hline Coagulopathy & $108(0.5)$ & $940(2.2)^{* *}$ & $106(0.6)$ & $228(1.2)^{* *}$ \\
\hline \multicolumn{5}{|l|}{ Pre-index clinical scores } \\
\hline Deyo-Charlson Comorbidity Index & $1.4 \pm 1.7$ & $2.2 \pm 2.3 * *$ & $1.5 \pm 1.8$ & $1.5 \pm 1.8$ \\
\hline $\mathrm{CHADS}_{2}$ & $1.6 \pm 1.2$ & $2.0 \pm 1.3^{* *}$ & $1.6 \pm 1.2$ & $1.6 \pm 1.2$ \\
\hline HAS-BLED & $1.4 \pm 1.0$ & $1.6 \pm 1.1 * *$ & $1.4 \pm 1.0$ & $1.4 \pm 1.0$ \\
\hline ATRIA & $1.5 \pm 1.6$ & $2.3 \pm 2.2 * *$ & $1.5 \pm 1.6$ & $1.7 \pm 1.7 * *$ \\
\hline Baseline total healthcare costs (\$US) & $23,266 \pm 34,208$ & $43,803 \pm 84,343 * *$ & $23,629 \pm 34,667$ & $29,479 \pm 56,106^{* *}$ \\
\hline
\end{tabular}

Data are presented as mean \pm standard deviation or $n(\%)$ unless otherwise indicated

$A F$ atrial fibrillation, $C O P D$ chronic obstructive pulmonary disease, $N V A F$ non-valvular atrial fibrillation

$* p<0.05, * * p<0.001$ dabigatran vs. warfarin cohorts

were aged $\geq 55$ years and mostly subscribed to the exclusive provider organization (EPO)/preferred provider organization (PPO) and comprehensive/indemnity health plan type (Table S2). Patients were predominantly from the Southern US census region (approximately 35\%) and male (approximately 63\%). The most prevalent comorbidities were hypertension $(68.1 \%)$, coronary heart disease (approximately $31 \%$ ), diabetes mellitus $(26.7 \%)$, psychiatric disorders (approximately 19.5\%), and HF (18.6\%). Dabigatran users were significantly less likely to have 
coagulopathy than were warfarin users ( 0.6 vs. $1.2 \%$, respectively; $p<0.01$ ). Both treatment cohorts had similar mean \pm SD clinical scores: DCCI $(1.5 \pm 1.8), \mathrm{CHADS}_{2}$ $(1.6 \pm 1.2)$, and HAS-BLED $(1.4 \pm 1.0)$. Dabigatran users had a significantly lower ATRIA score than did warfarin users $(1.5 \pm 1.6$ vs. $1.7 \pm 1.7$, respectively; $p<0.01)$. The other medications most commonly used during the pre-index period were beta blockers (approximately 57\%), antihyperlipidemics (approximately 55\%), and diuretics (43\%). Mean total healthcare costs during the pre-index period were significantly lower for dabigatran users than for warfarin users (\$US23,629 vs. 29,479, respectively; $p<0.01)$.

\subsection{All-cause Healthcare Expenditures, Per Patient Per Month (PPPM)}

Overall, the unadjusted all-cause total PPPM healthcare expenditures were significantly lower for patients treated with dabigatran than for those treated with warfarin (\$US3094.15 vs. 3479.03 , respectively; $p<0.001$ ). Not surprisingly, the mean outpatient pharmacy costs for dabigatran users were significantly higher than those for warfarin users (\$US563.07 vs. 349.83, respectively; $p<0.001$ ), possibly due to substantial price differences between dabigatran and warfarin. Dabigatran users had significantly lower inpatient costs (\$US916.20 vs. 1210.74 ), total outpatient costs (\$US1614.88 vs. 1919.45), emergency room visit costs (\$US57.84 vs. 71.51), outpatient office costs (\$US131.66 vs. 157.30), and other outpatient costs (\$US1425.38 vs. 1690.65) than did warfarin users ( $p<0.001$ for all values). Of note, higher costs in the warfarin cohort were primarily driven by inpatient admission and outpatient services (Fig. S1).

After controlling for demographic and clinical characteristics, multivariate results were consistent with these descriptive results and confirmed that costs for dabigatran users were significantly lower than those for warfarin users (Tables 2,3). After adjusting for covariates, the all-cause costs PPPM for healthcare, inpatient stays, and outpatient services were significantly lower for patients newly treated with dabigatran than for those newly treated with warfarin (\$US3053.44 vs. $3433.25 ;$ \$US904.30 vs. 1194.00; \$US1594.63 vs. 1894.20 , respectively; all $p<0.001$ ). In contrast, the average PPPM costs for outpatient pharmacy were higher for dabigatran users than for warfarin users (\$US556.67 vs. 345.23 , respectively; $p<0.001$ ).

\subsection{Stroke-Specific Healthcare Expenditures, PPPM}

Although the prevalence of stroke-specific events was low among both groups during the up to 12-month follow-up period, the rate was significantly lower among dabigatran users than among warfarin users (87 [0.5\%] vs. 142 [0.8\%] patient, respectively; $p<0.001)$. Stroke-specific PPPM healthcare costs were significantly lower for dabigatran users than for warfarin users (\$US30.37 vs. 40.99, respectively; $p<0.001$ ) (Fig. S2 in the ESM). In addition, PPPM outpatient costs, outpatient office visits, and other outpatient service costs were significantly lower for dabigatran users than for warfarin users (\$US7.36 vs. 12.20, \$US1.18 vs. 0.72 , and \$US5.96 vs. 10.43 , respectively; $p<0.05$ for all values). No significant differences were observed for stroke-specific inpatient and emergency room costs between dabigatran and warfarin users (\$US23.00 vs. 28.80 and $\$$ US0.68 vs. 0.59 , respectively; $p>0.05$ for both values). Sensitivity analyses revealed one outlier dabigatran patient with stroke-specific inpatient costs of \$US479,297 for one visit. When this patient was excluded, stroke-specific inpatient costs were significantly lower for dabigatran users than for warfarin users (\$US11.57 vs. 28.80 , respectively; $p<0.01$ ).

\subsection{Bleed-Specific Healthcare Expenditures, PPPM}

The total number of bleed-specific events was significantly lower among dabigatran users than among warfarin users (227 [1.2\%] vs. 294 [1.6\%] patients, respectively; $p=0.003$ ). Similar to all-cause and stroke-specific healthcare expenditures, bleed-specific total healthcare costs PPPM were significantly lower for dabigatran than for warfarin users ( $\$$ US50.00 vs. 73.49 , respectively; $p<0.001$ ) (Fig. S3 in the ESM). In addition, inpatient and emergency room costs were significantly lower for dabigatran than for warfarin users (\$US27.75 vs. 48.66 and \$US1.97 vs. 3.92, respectively; $p<0.01$ for both values). There were no significant differences in total outpatient, outpatient office, and other outpatient costs between dabigatran and warfarin users (\$US22.25 vs. 24.83 , \$US1.81 vs. 1.83 , and $\$ \mathrm{US} 18.47$ vs. 19.08 , respectively; $p>0.05$ for all values). Sensitivity analyses revealed one outlier patient receiving warfarin with bleed-specific inpatient costs of \$US309,011 for one visit. When this patient was excluded, bleed-specific inpatient costs remained significantly lower for dabigatran than for warfarin patients (\$US27.75 vs. 48.66 , respectively; $p<0.01$ ).

\section{Discussion}

In this retrospective study, patients newly diagnosed with NVAF and newly treated with dabigatran had considerably lower PPPM all-cause total, inpatient, and outpatient costs than matched patients treated with warfarin. In addition, patients newly treated with dabigatran had significantly lower stroke-specific total healthcare costs and total 
Table 2 Generalized linear model results for all-cause total and inpatient expenditures

\begin{tabular}{|c|c|c|c|c|}
\hline Variables & Total healthcare costs & $p$ value & Inpatient costs & $p$ value \\
\hline Dabigatran vs. warfarin & $0.92(0.90-0.94)$ & $<0.0001$ & $0.72(0.68-0.77)$ & $<0.0001$ \\
\hline \multicolumn{5}{|l|}{ Age group (years) } \\
\hline \multicolumn{5}{|l|}{$<65$ (reference) } \\
\hline $65-74$ & $0.70(0.67-0.73)$ & $<0.0001$ & $0.81(0.73-0.90)$ & $<0.0001$ \\
\hline$\geq 75$ & $0.74(0.72-0.77)$ & $<0.0001$ & $0.75(0.68-0.82)$ & $<0.0001$ \\
\hline Sex (male) & $0.93(0.91-0.95)$ & $<0.0001$ & $0.95(0.89-1.01)$ & 0.090 \\
\hline \multicolumn{5}{|l|}{ Health plan type } \\
\hline \multicolumn{5}{|l|}{ Comprehensive/indemnity (reference) } \\
\hline $\mathrm{EPO} / \mathrm{PPO}$ & $1.09(1.03-1.14)$ & 0.002 & $1.05(0.91-1.21)$ & 0.510 \\
\hline POS with and without capitation & $1.15(1.10-1.20)$ & $<0.0001$ & $1.27(1.13-1.42)$ & $<0.0001$ \\
\hline HMO & $1.32(1.28-1.36)$ & $<0.0001$ & $1.38(1.28-1.49)$ & $<0.0001$ \\
\hline CDHP/HDHP/other/unknown & $1.18(1.13-1.24)$ & $<0.0001$ & $0.97(0.85-1.11)$ & 0.705 \\
\hline \multicolumn{5}{|l|}{ Region } \\
\hline \multicolumn{5}{|l|}{ North east (reference) } \\
\hline West & $1.00(0.96-1.03)$ & 0.790 & $0.87(0.78-0.96)$ & 0.005 \\
\hline South/unknown & $0.93(0.90-0.96)$ & $<0.0001$ & $0.77(0.71-0.84)$ & $<0.0001$ \\
\hline North central & $0.89(0.86-0.92)$ & $<0.0001$ & $0.77(0.70-0.83)$ & $<0.0001$ \\
\hline Deyo-Charlson Comorbidity Index & $1.07(1.06-1.08)$ & $<0.0001$ & $1.01(0.99-1.04)$ & 0.418 \\
\hline Number of unique generic drugs prescribed & $1.02(1.02-1.02)$ & $<0.0001$ & $1.02(1.01-1.03)$ & $<0.0001$ \\
\hline Number of inpatient stays & $0.95(0.94-0.97)$ & $<0.0001$ & $0.98(0.94-1.03)$ & 0.383 \\
\hline Number of office visits & $1.01(1.01-1.01)$ & $<0.0001$ & $1.00(1.00-1.01)$ & 0.143 \\
\hline \multicolumn{5}{|l|}{ Diagnosis in the pre-index period (yes or no) } \\
\hline Hip fracture & $1.11(0.96-1.28)$ & 0.151 & $1.03(0.70-1.50)$ & 0.896 \\
\hline Chronic kidney disease & $1.08(1.02-1.13)$ & 0.004 & $1.25(1.09-1.43)$ & 0.001 \\
\hline COPD & $0.96(0.93-0.99)$ & 0.015 & $1.15(1.05-1.25)$ & 0.002 \\
\hline Cirrhosis or hepatitis & $1.03(0.94-1.14)$ & 0.495 & $1.13(0.87-1.46)$ & 0.351 \\
\hline CAD & $1.03(1.00-1.06)$ & 0.044 & $1.10(1.02-1.18)$ & 0.012 \\
\hline Diabetes & $0.92(0.88-0.97)$ & 0.001 & $0.97(0.85-1.11)$ & 0.680 \\
\hline Heart failure & $1.13(1.08-1.18)$ & $<0.0001$ & $1.24(1.11-1.39)$ & 0.000 \\
\hline Hypertension & $0.92(0.88-0.96)$ & 0.000 & $0.89(0.79-1.00)$ & 0.049 \\
\hline Myocardial infarction (acute or old) & $0.93(0.89-0.98)$ & 0.004 & $0.98(0.86-1.12)$ & 0.740 \\
\hline Paraplegic or hemiplegic & $0.98(0.89-1.07)$ & 0.620 & $1.00(0.78-1.29)$ & 0.993 \\
\hline Psychiatric disorders & $1.07(1.04-1.10)$ & $<0.0001$ & $1.10(1.02-1.18)$ & 0.019 \\
\hline Venous thrombosis & $1.02(0.94-1.11)$ & 0.654 & $1.10(0.87-1.39)$ & 0.445 \\
\hline Ischemic or hemorrhagic bleed & $0.95(0.88-1.02)$ & 0.188 & $1.07(0.88-1.30)$ & 0.499 \\
\hline Intracranial, extracranial, or GI bleed & $1.00(0.96-1.04)$ & 0.925 & $1.04(0.93-1.15)$ & 0.494 \\
\hline $\mathrm{CHADS}_{2}$ score & $0.95(0.93-0.98)$ & 0.001 & $0.96(0.89-1.04)$ & 0.281 \\
\hline HAS-BLED score & $0.98(0.96-1.00)$ & 0.077 & $0.97(0.91-1.02)$ & 0.250 \\
\hline Log (total cost in the pre-index period) & $1.14(1.13-1.15)$ & $<0.0001$ & $1.15(1.12-1.18)$ & $<0.0001$ \\
\hline Days from first $\mathrm{AF}$ diagnosis to index date & $1.00(1.00-1.00)$ & $<0.0001$ & $1.00(1.00-1.00)$ & 0.058 \\
\hline \multicolumn{5}{|l|}{ Prescription (yes or no) } \\
\hline Beta blockers & $0.96(0.93-0.98)$ & 0.000 & $0.95(0.90-1.02)$ & 0.152 \\
\hline Calcium channel blockers & $1.04(1.02-1.07)$ & 0.001 & $1.09(1.02-1.16)$ & 0.011 \\
\hline Diuretics & $0.96(0.94-0.99)$ & 0.002 & $1.02(0.95-1.09)$ & 0.550 \\
\hline Other antihypertensives & $0.95(0.92-0.97)$ & $<0.0001$ & $0.99(0.93-1.06)$ & 0.839 \\
\hline Antihyperlipidemics & $0.91(0.88-0.93)$ & $<0.0001$ & $0.93(0.87-0.99)$ & 0.023 \\
\hline Corticosteroids & $1.01(0.98-1.04)$ & 0.450 & $0.98(0.92-1.05)$ & 0.658 \\
\hline Antidiabetics & $1.01(0.97-1.06)$ & 0.506 & $1.16(1.04-1.30)$ & 0.011 \\
\hline
\end{tabular}


Table 2 continued

\begin{tabular}{|c|c|c|c|c|}
\hline Variables & Total healthcare costs & $p$ value & Inpatient costs & $p$ value \\
\hline Anti-arrhythmics & $1.23(1.20-1.27)$ & $<0.0001$ & $1.06(0.97-1.15)$ & 0.197 \\
\hline Ketoconazole & $0.98(0.91-1.06)$ & 0.668 & $1.11(0.90-1.37)$ & 0.347 \\
\hline Antiplatelets & $1.08(1.04-1.13)$ & $<0.0001$ & $1.22(1.10-1.36)$ & 0.000 \\
\hline \multicolumn{5}{|l|}{ Predicted costs (\$US) } \\
\hline Dabigatran & 3053 (2968-3138) & $<0.0001$ & $904(837-972)$ & $<0.0001$ \\
\hline Warfarin & 3433 (3308-3138) & $\operatorname{Ref}^{1}$ & 1194 (1123-1265) & $\operatorname{Ref}^{1}$ \\
\hline
\end{tabular}

$A F$ atrial fibrillation, $C A D$ coronary artery disease, $C D H P$ consumer-driven health plan, $C O P D$ chronic obstructive pulmonary disease, $E P O$ exclusive provider organization, $G I$ gastrointestinal, $H D H P$ high-deductible health plan, $H M O$ health maintenance organization, $P O S$ point-ofservice plan, $P P O$ preferred provider organization

${ }^{\text {a }}$ Reference group

outpatient costs and bleed-specific total healthcare costs and inpatient costs compared with those treated with warfarin. Understanding the magnitude of the resource and cost burden associated with treatment may provide useful insights when selecting the optimal therapeutic option for patients with NVAF. Inpatient hospitalization costs are a major factor responsible for incremental economic burden among NVAF patients treated with warfarin and dabigatran $[20,21,36]$. Our findings demonstrated that approximately 30 and $35 \%$, respectively, of total healthcare costs were due to inpatient costs among dabigatran and warfarin users. Previous work corroborated by healthcare resource (HCRU) findings using the same dataset and subset of patients (presented elsewhere) supports these findings [36]. Recent studies reporting costs in patients newly diagnosed with NVAF and treated with dabigatran or warfarin produced results consistent with those of the present study $[20,21]$. A retrospective analysis of patients with NVAF treated with dabigatran or warfarin demonstrated that, despite higher pharmacy costs for dabigatran versus warfarin ( $\$$ US10,007 vs. 5647, respectively), the overall healthcare costs during a 12-month follow-up period were significantly lower for dabigatran than for warfarin users ( $\$$ US17,617 vs. 19,557, respectively) [21]. In contrast, other studies have reported that, while NOACs are associated with greater quality-adjusted life expectancy than is warfarin, they may not represent good value for money depending on patients' willingness to pay [37]. Several factors may potentially explain the lower costs in the current analysis compared with previous studies. First, the sample size in our study was substantially higher than in previous studies $(18,980$ vs. 1102 and 869 patients per treatment arm) [20, 21]. Second, the study population differed from that studied by Canestaro et al. [37]. These authors utilized a hypothetical cohort of patients aged 70 years with an average $\mathrm{CHADS}_{2}$ score of 2 who were eligible for treatment with warfarin, their data were limited to that from clinical trials (compared with claims data), and they reported incremental cost-effectiveness ratios (ICERs) from their economic model (compared with direct total costs including medical and pharmacy costs).

In addition, stroke-specific total costs and total outpatient costs, and bleed-specific total costs and inpatient costs, were significantly lower for dabigatran than for warfarin users. These findings are supported by the significantly lower stroke- and bleed-specific events among dabigatran users than among warfarin users [36]. In addition, patients newly treated with dabigatran had significantly lower mean stroke-specific healthcare utilization (e.g., 45\% fewer inpatient admissions [including shorter length of stay] and $25 \%$ fewer outpatient visits) and significantly lower bleed-specific utilization (e.g., $33 \%$ fewer inpatient admissions [including shorter length of stay] and $13 \%$ fewer outpatient visits) than did warfarin users [36]. The mean stroke- and bleed-specific costs (\$US30 and 50 PPPM, respectively) for newly diagnosed patients receiving dabigatran in our analysis were comparable with results reported by Francis et al. [21] at 3 months (\$US144 and 148 , respectively). However, mean stroke- and bleedspecific costs for newly diagnosed patients receiving warfarin in our analysis were higher (\$US41 and 73 vs. \$US77 and 84 PPPM at 3 months, respectively). While they found no significant differences in hospital costs and resource utilization attributable to ischemic stroke or bleeding, their study was not powered to detect a difference in driverspecific utilization and costs [21]. Although our study demonstrates significant differences in costs among several stroke- and bleed-specific cost categories, future studies with larger sample sizes are necessary to confirm our findings.

Several limitations inherent to all retrospective claimsbased cohort studies also apply to this study. However, as both treatment groups compared in this study were selected from the same database, many of the limitations apply to both groups, thus reducing any potential bias. First, there is the potential for misclassification of NVAF, covariates, and 
Table 3 Generalized linear model results for all-cause total outpatient and outpatient pharmacy expenditures

\begin{tabular}{|c|c|c|c|c|}
\hline Variables & Total outpatient costs & $p$ value & Outpatient pharmacy costs & $p$ value \\
\hline Dabigatran vs. warfarin & $0.88(0.86-0.90)$ & $<0.0001$ & $1.82(1.80-1.85)$ & $<0.0001$ \\
\hline \multicolumn{5}{|l|}{ Age group } \\
\hline \multicolumn{5}{|l|}{$<65$ years (reference) } \\
\hline $65-74$ years & $0.64(0.61-0.67)$ & $<0.0001$ & $0.90(0.88-0.93)$ & $<0.0001$ \\
\hline$\geq 75+$ years & $0.73(0.70-0.75)$ & $<0.0001$ & $0.96(0.94-0.98)$ & 0.001 \\
\hline Sex (male) & $0.89(0.87-0.92)$ & $<0.0001$ & $0.94(0.92-0.95)$ & $<0.0001$ \\
\hline \multicolumn{5}{|l|}{ Health plan type } \\
\hline \multicolumn{5}{|l|}{ Comprehensive/indemnity (reference) } \\
\hline $\mathrm{EPO} / \mathrm{PPO}$ & $1.13(1.07-1.19)$ & $<0.0001$ & $0.98(0.94-1.01)$ & 0.210 \\
\hline POS with and without capitation & $1.06(1.02-1.11)$ & 0.007 & $0.89(0.86-0.91)$ & $<0.0001$ \\
\hline HMO & $1.34(1.30-1.38)$ & $<0.0001$ & $0.96(0.94-0.98)$ & $<0.0001$ \\
\hline CDHP/HDHP/other/unknown & $1.24(1.18-1.31)$ & $<0.0001$ & $0.93(0.90-0.97)$ & $<0.0001$ \\
\hline \multicolumn{5}{|l|}{ Region } \\
\hline \multicolumn{5}{|l|}{ North east (reference) } \\
\hline West & $1.10(1.05-1.14)$ & $<0.0001$ & $0.92(0.90-0.95)$ & $<0.0001$ \\
\hline South/unknown & $1.03(0.99-1.06)$ & 0.108 & $0.96(0.94-0.98)$ & $<0.0001$ \\
\hline North central & $0.95(0.92-0.98)$ & 0.004 & $0.93(0.91-0.95)$ & $<0.0001$ \\
\hline Deyo-Charlson Comorbidity Index & $1.09(1.08-1.10)$ & $<0.0001$ & $1.07(1.06-1.08)$ & $<0.0001$ \\
\hline Number of unique generic drugs prescribed & $1.01(1.01-1.02)$ & $<0.0001$ & $1.05(1.05-1.05)$ & $<0.0001$ \\
\hline Number of inpatient stays & $0.96(0.94-0.97)$ & $<0.0001$ & $0.91(0.90-0.93)$ & $<0.0001$ \\
\hline Number of office visits & $1.01(1.01-1.01)$ & $<0.0001$ & $1.00(1.00-1.00)$ & 0.576 \\
\hline \multicolumn{5}{|l|}{ Diagnosis in the pre-index period (yes or no) } \\
\hline Hip fracture & $1.22(1.05-1.42)$ & 0.011 & $0.93(0.84-1.03)$ & 0.151 \\
\hline Chronic kidney disease & $1.12(1.06-1.18)$ & $<0.0001$ & $0.97(0.94-1.01)$ & 0.133 \\
\hline COPD & $0.90(0.87-0.93)$ & $<0.0001$ & $1.02(0.99-1.04)$ & 0.186 \\
\hline Cirrhosis or hepatitis & $1.03(0.93-1.14)$ & 0.527 & $1.14(1.06-1.22)$ & 0.000 \\
\hline CAD & $1.01(0.99-1.04)$ & 0.350 & $0.99(0.97-1.01)$ & 0.404 \\
\hline Diabetes & $0.88(0.83-0.93)$ & $<0.0001$ & $1.01(0.97-1.04)$ & 0.720 \\
\hline Heart failure & $1.03(0.99-1.08)$ & 0.157 & $0.94(0.92-0.97)$ & $<0.0001$ \\
\hline Hypertension & $0.91(0.86-0.95)$ & $<0.0001$ & $1.06(1.03-1.10)$ & $<0.0001$ \\
\hline Myocardial infarction (acute or old) & $0.90(0.86-0.95)$ & $<0.0001$ & $0.92(0.89-0.95)$ & $<0.0001$ \\
\hline Paraplegic or hemiplegic & $1.02(0.92-1.13)$ & 0.745 & $0.89(0.84-0.96)$ & 0.001 \\
\hline Psychiatric disorders & $1.00(0.97-1.03)$ & 0.811 & $0.98(0.97-1.00)$ & 0.119 \\
\hline Venous thrombosis & $0.89(0.81-0.98)$ & 0.016 & $1.13(1.06-1.20)$ & $<0.0001$ \\
\hline Ischemic or hemorrhagic bleed & $0.89(0.82-0.97)$ & 0.005 & $0.99(0.94-1.04)$ & 0.630 \\
\hline Intracranial, extracranial, or GI bleed & $1.01(0.97-1.06)$ & 0.490 & $0.95(0.93-0.98)$ & 0.001 \\
\hline $\mathrm{CHADS}_{2}$ score & $0.98(0.95-1.01)$ & 0.252 & $0.97(0.95-0.99)$ & 0.001 \\
\hline HAS-BLED score & $0.97(0.95-0.99)$ & 0.011 & $0.97(0.95-0.98)$ & $<0.0001$ \\
\hline Log (total cost in the pre-index period) & $1.13(1.12-1.14)$ & $<0.0001$ & $1.16(1.15-1.17)$ & $<0.0001$ \\
\hline Days from first $\mathrm{AF}$ diagnosis to index date & $1.00(1.00-1.00)$ & $<0.0001$ & $1.00(1.00-1.00)$ & 0.000 \\
\hline \multicolumn{5}{|l|}{ Prescription (yes or no) } \\
\hline Beta blockers & $0.96(0.94-0.99)$ & 0.002 & $0.91(0.89-0.92)$ & $<0.0001$ \\
\hline Calcium channel blockers & $0.99(0.96-1.01)$ & 0.337 & $0.97(0.96-0.99)$ & 0.002 \\
\hline Diuretics & $0.91(0.89-0.94)$ & $<0.0001$ & $0.99(0.97-1.00)$ & 0.125 \\
\hline Other antihypertensives & $0.93(0.91-0.96)$ & $<0.0001$ & $0.90(0.89-0.92)$ & $<0.0001$ \\
\hline Antihyperlipidemics & $0.89(0.87-0.91)$ & $<0.0001$ & $1.08(1.06-1.10)$ & $<0.0001$ \\
\hline Corticosteroids & $1.03(1.01-1.06)$ & 0.014 & $0.97(0.95-0.99)$ & 0.001 \\
\hline Antidiabetics & $0.92(0.88-0.96)$ & 0.000 & $1.12(1.08-1.15)$ & $<0.0001$ \\
\hline
\end{tabular}


Table 3 continued

\begin{tabular}{lclll}
\hline Variables & Total outpatient costs & $p$ value & Outpatient pharmacy costs & $p$ value \\
\hline Anti-arrhythmics & $1.39(1.35-1.44)$ & $<0.0001$ & $0.96(0.94-0.98)$ & $0.93(0.88-0.98)$ \\
Ketoconazole & $1.01(0.93-1.10)$ & 0.766 & $1.03(1.00-1.06)$ & 0.011 \\
Antiplatelets & $1.01(0.97-1.05)$ & 0.698 & & 0.045 \\
Predicted costs (\$US) & & & & \\
Dabigatran & $1594(1549-1638)$ & $<0.0001$ & $356(458-563)$ & $<0.0001$ \\
Warfarin & $1894(1798-1991)$ & Ref $^{\mathrm{a}}$ & $345-357)$ & Ref $^{\mathrm{a}}$ \\
\hline
\end{tabular}

Data are presented as mean estimate (95\% confidence interval)

$A F$ atrial fibrillation, $C A D$ coronary artery disease, $C D H P$ consumer-driven health plan, $C O P D$ chronic obstructive pulmonary disease, $E P O$ exclusive provider organization, GI gastrointestinal, $H D H P$ high-deductible health plan, $H M O$ health maintenance organization, $P O S$ point-ofservice plan, $P P O$ preferred provider organization

${ }^{a}$ Reference group

outcomes because data for this study were derived from administrative claims, which are subject to data coding limitations and data entry error. Second, medication exposure was based on filled prescriptions. We assumed these patients took medications as prescribed, but it was not possible to confirm this assumption. Third, there may be systematic differences between the two cohorts that could partially account for differences found in healthcare costs, for example, the dabigatran cohort had significantly lower baseline costs than the warfarin cohort. However, we matched on the logarithm of total baseline costs and controlled for baseline costs in the multivariate analyses to make the predicted costs comparable between the two cohorts. Given that this study was retrospective in nature, unknown confounders may have influenced stroke- or bleed-related events, thereby affecting the cost results. Fourth, given the low sample size of patients with stroke-specific and bleedspecific events, multivariate analyses were not performed on these outcomes. Future research should explore eventspecific costs and utilization using a much larger study population to corroborate our findings. Fifth, stroke- and bleed-specific hospitalizations were identified using diagnosis codes only, and disease-related groups were not used to identify such hospitalizations. This might have led to underestimation of costs. Sixth, this study consisted of individuals with commercial or private Medicare supplemental coverage, thereby limiting the generalizability of the findings to patients with NVAF with other insurance or those without health insurance coverage. Finally, the present study did not compare other NOACs (i.e., apixaban and rivaroxaban) with warfarin or dabigatran. Our ongoing research (presented elsewhere) has compared these OACs to assess all-cause and stroke- and bleed-specific HCRU and cost.

Despite these limitations, the potential strengths of this study include that it evaluated a large sample of patients from two large nationally representative US claims databases (commercial and Medicare Supplemental). In addition, compared with previous claims-based studies $[9,20,21]$, this analysis provides more recent data on allcause and event-specific (i.e., stroke and bleed) costs among patients with NVAF newly treated with dabigatran or warfarin. Propensity score matching was applied to generate comparable cohorts with similar baseline demographic and clinical characteristics. Furthermore, compared with previous work, which was not powered to detect a difference in clinical and economic outcomes between dabigatran and warfarin users [21], this study was designed to detect potential differences among stroke- and bleedspecific costs in patients with NVAF across dabigatran and warfarin users. Although dabigatran has been on the market for over 5 years, data directly comparing the stroke- and bleed-specific costs for dabigatran versus warfarin are limited. Given the benefits of NOACs, it is important to investigate all-cause and event-specific outcomes that will provide valuable information for the medical community. Results from this study will assist US health providers, health plans, and patients in assessing the comparative effectiveness in economic terms among patients with newly diagnosed NVAF and newly treated with anticoagulant therapy to reduce stroke risk.

\section{Conclusion}

The results of this study suggest that patients with NVAF newly treated with dabigatran were associated with significantly lower all-cause total healthcare costs compared with warfarin-treated patients. Furthermore, patents treated with dabigatran had significantly lower stroke-specific and bleed-specific total costs. Despite the higher pharmacy cost differential of dabigatran relative to warfarin, potential cost savings may be obtained with the use of dabigatran over warfarin due to lower all-cause inpatient, outpatient, and driver-specific costs. 
Acknowledgements Editorial/writing assistance for this manuscript was provided by Dr. Santosh Tiwari, who was compensated by Truven Health Analytics, an IBM Company.

\section{Compliance with Ethical Standards}

Funding This study was funded by Boehringer-Ingelheim Pharmaceuticals Inc. and conducted by Truven Health Analytics, USA.

Conflict of interest All listed authors meet the criteria for authorship set forth by the International Committee for Medical Journal Editors. Adrienne Gilligan, Xue Song, Caroline Henriques, and David Smith are employees of Truven Health Analytics, which received compensation from Boehringer-Ingelheim Pharmaceuticals Inc. for the overall conduct of the study and preparation of this manuscript. Pranav Gandhi, Cheng Wang, and Stephen Sander are employees of Boehringer-Ingelheim Pharmaceuticals Inc.

Open Access This article is distributed under the terms of the Creative Commons Attribution-NonCommercial 4.0 International License (http://creativecommons.org/licenses/by-nc/4.0/), which permits any noncommercial use, distribution, and reproduction in any medium, provided you give appropriate credit to the original author(s) and the source, provide a link to the Creative Commons license, and indicate if changes were made.

\section{References}

1. Centers for Disease Control and Prevention. Division for Heart Disease and Stroke Prevention. Atrial fibrillation fact sheet. Atlanta, GA: U.S. Department of Health \& Human Services; 2015. https://www.cdc.gov/dhdsp/data_statistics/fact_sheets/fs_ atrial_fibrillation.htm. Accessed 12 Sept 2016.

2. Dasta JF, Pilon D, Mody SH, Lopatto J, Laliberté F, Germain G, et al. Daily costs of hospitalization in non-valvular atrial fibrillation patients treated with anticoagulant therapy. J Med Econ. 2015;18(12):1041-9. doi:10.3111/13696998.2015.1074583.

3. Shea JB, Sears SF. A patient's guide to living with atrial fibrillation. Circulation. 2008;117(20):e340-3. doi:10.1161/ CIRCULATIONAHA.108.780577.

4. Go AS, Hylek EM, Phillips KA, Chang Y, Henault LE, Selby JV, et al. Prevalence of diagnosed atrial fibrillation in adults: national implications for rhythm management and stroke prevention: the AnTicoagulation and Risk Factors in Atrial Fibrillation (ATRIA) Study. JAMA. 2001;285(18):2370-5.

5. Wolf PA, D'Agostino RB, Belanger AJ, Kannel WB. Probability of stroke: a risk profile from the Framingham Study. Stroke. 1991;22(3):312-8.

6. Deplanque D, Leys D, Parnetti L, Schmidt R, Ferro J, De Reuck J, et al. Stroke prevention and atrial fibrillation: reasons leading to an inappropriate management. Main results of the SAFE II study. Br J Clin Pharmacol. 2004;57(6):798-806. doi:10.1111/j.13652125.2004.02086.x.

7. Mozaffarian D, et al. Heart disease and stroke statistics—2015 update: a report from the American Heart Association [published errata appear in Circulation 2015;131(24):e535 and Circulation 2015;133(8):e417). Circulation. 2015;131(4):E29-322.

8. Fitch K, Broulette J, Kwong WJ. The economic burden of ischemic stroke and major hemorrhage in medicare beneficiaries with nonvalvular atrial fibrillation: a retrospective claims analysis. Am Health Drug Benefits. 2014;7(4):200.

9. Mercaldi CJ, Siu K, Sander SD, Walker DR, Wu Y, Li Q, et al. Long-term costs of ischemic stroke and major bleeding events among Medicare patients with nonvalvular atrial fibrillation. Cardiol Res Pract. 2012;2012:645469. doi:10.1155/2012/645469.

10. January CT, Wann LS, Alpert JS, Calkins H, Cigarroa JE, Cleveland JC Jr, et al. 2014 AHA/ACC/HRS guideline for the management of patients with atrial fibrillation: executive summary: a report of the American College of Cardiology/American Heart Association Task Force on practice guidelines and the Heart Rhythm Society. Circulation. 2014;130(23):2071-104. doi:10.1161/cir.0000000000000040.

11. Camm AJ, Lip GY, De Caterina R, Savelieva I, Atar D, Hohnloser SH, et al. 2012 focused update of the ESC Guidelines for the management of atrial fibrillation: an update of the 2010 ESC Guidelines for the management of atrial fibrillation. Developed with the special contribution of the European Heart Rhythm Association. Eur Heart J. 2012;33(21):2719-47. doi:10.1093/ eurheartj/ehs253.

12. You JJ, Singer DE, Howard PA, Lane DA, Eckman MH, Fang MC, et al. Antithrombotic therapy for atrial fibrillation: antithrombotic therapy and prevention of thrombosis: American College of Chest Physicians evidence-based clinical practice guidelines. Chest J. 2012;141(2_suppl):e531S-e75S. doi:10. 1378/chest.11-2304.

13. Wann LS, Curtis AB, Ellenbogen KA, Estes NA 3rd, Ezekowitz MD, Jackman WM, et al. 2011 ACCF/AHA/HRS focused update on the management of patients with atrial fibrillation (update on Dabigatran): a report of the American College of Cardiology Foundation/American Heart Association Task Force on practice guidelines. Circulation. 2011;123(10):1144-50. doi:10.1161/CIR. 0b013e31820f14c0.

14. Enterzari-Maleki T, Dousti S, Hamishehkar H, Gholami K. A systematic review on comparing 2 common methods for management of warfarin therapy: pharmacist-led service versus usual medical care. J Clin Pharmacol. 2016;56(1):24-38.

15. Connolly SJ, Ezekowitz MD, Yusuf S, Eikelboom J, Oldgren J, Parekh A, Pogue J, Reilly PA, Themeles E, Varrone J, Wang S, Alings M, Xavier D, Zhu J, Diaz R, Lewis BS, Darius H, Diener HC, Joyner CD, Wallentin L, Committee R-LS Investigators. Dabigatran versus warfarin in patients with atrial fibrillation. N Engl J Med. 2009;361:1139-51.

16. Connolly SJ, Wallentin L, Yusuf S. Additional events in the RELY trial. N Engl J Med. 2014;371:1464-5.

17. Pisters R, Lane DA, Nieuwlaat R, de Vos CB, Crijns HJ, Lip GY. A novel user-friendly score (HAS-BLED) to assess 1-year risk of major bleeding in patients with atrial fibrillation: the Euro Heart Survey. Chest. 2010;138:1093-100.

18. Graham DJ, Reichman ME, Wernecke M, Zhang R, Southworth MR, Levenson M, Sheu TC, Mott K, Goulding MR, Houstoun M, MaCurdy TE, Worrall C, Kelman JA. Cardiovascular, bleeding, and mortality risks in elderly Medicare patients treated with dabigatran or warfarin for nonvalvular atrial fibrillation. Circulation. 2015;131:157-64.

19. Sarich TC, Seltzer JH, Berkowitz SD, Costin' J, Curnutte JT, Gibson CM, Hoffman M, Kaminskas E, Krucoff MW, Levy JH, Mintz PD, Reilly PA, Sager PT, Singer DE, Stockbridge N, Weitz JI, Kowey PR. Novel oral anticoagulants and reversal agents: Considerations for clinical development. Am Heart J. 2015;169(6):751-7.

20. Bancroft T, Lim J, Wang C, Sander SD, Swindle JP. Health care resource utilization, costs, and persistence in patients newly diagnosed as having nonvalvular atrial fibrillation and newly treated with dabigatran versus warfarin in the United States. Clin Ther. 2016;38(3):545-56. e6. doi:10.1016/j.clinthera.2016.01. 008.

21. Francis K, Yu C, Alvrtsyan H, Sander S, Ghosh S, Rao Y, et al. Healthcare utilization and costs associated with dabigatran compared to warfarin treatment in newly diagnosed patients with 
non-valvular atrial fibrillation. Curr Med Res Opin. 2015;31(12):2189-95. doi:10.1185/03007995.2015.1092124.

22. Connolly SJ, Ezekowitz MD, Yusuf S, et al. Dabigatran versus warfarin in patients with atrial fibrillation. $\mathrm{N}$ Engl J Med. 2009;361:1139-51 (Erratum, N Engl J Med 2010;363:1877).

23. Connolly SJ, Ezekowitz MD, Yusuf S, Reilly PA, Wallentin L. Newly identified events in the RE-LY trial. N Engl J Med. 2010;363:1875-6.

24. Villines TC, Schnee J, Fraeman K, Siu K, Reynolds MW, Collins J, Schwartzman E. A comparison of the safety and effectiveness of dabigatran and warfarin in non-valvular atrial fibrillation patients in a large healthcare system. Thromb Haemost. 2015;114:1290-8.

25. Seeger JD, Bykov K, Bartels DB, Huybrechts K, Zint K, Schneeweiss $S$. Safety and effectiveness of dabigatran and warfarin in routine care of patients with atrial fibrillation. Thromb Haemost. 2015;114:1277-89.

26. Romanelli RJ, Nolting L, Doginsky M, Kym E, Orrico KB. Dabigatrans warfarin for atrial fibrillation in real-world clinical practice: a systematic review and meta-analysis. Circ Cardovasc Qual outcomes. 2016;9:126-34.

27. Larsen TB, Rasmussen LH, Skjøth F, Due KM, Callréus T, Rosenzweig M, et al. Efficacy and safety of dabigatran etexilate and warfarin in "real-world" patients with atrial fibrillation: a prospective nationwide cohort study. J Am Coll Cardiol. 2013;61(22):2264-73. doi:10.1016/j.jacc.2013.03.020.

28. Mucha L, Masia NA, Azelsen KJ. Per-patient-per-month drug costs in medicare part protected classes. Pharmacoeconomics. 2006;24(3):79-84.

29. Farr AM, Johnston SS, Ritchings C, Brouillette M, et al. Persistence, adherence, and all-cause healthcare costs in atazanavirand darunavir-treated patients with human immunodeficiency virus in a real-world setting. J Med Econ. 2016;19(4):386-96.
30. Hao Y, Li N, Fang AP, Koo V, et al. Real-world analysis of medical costs and healthcare resource utilization in elderly women with $\mathrm{HR}+/ \mathrm{HER} 2$-metastatic breast cancer receiving everolimus-based therapy or chemotherapy. Adv Ther. 2016;33(6):983-97.

31. Palmer JB, Li Y, Herrera V, Liao M, et al. Treatment patterns and costs for anti-TNF $\alpha$ biologic therapy in patients with psoriatic arthritis. BMC Musculoskelet Disord. 2016;. doi:10.1186/ s12891-016-1102-z.

32. Vekeman F, Sasane M, Cheng WY, Ramanakumar AV, et al. Adherence to iron chelation therapy and associated healthcare resource utilization and costs in Medicaid patients with sickle cell disease and thalassemia. J Med Econ. 2016;19(3):292-303.

33. Anderson RJ. Cost analysis of a managed care decentralized outpatient pharmacy anticoagulation service. J Manag Care Pharm. 2004;10(2):159-65.

34. Gage BF, Waterman AD, Shannon W, Boechler M, Rich MW, Radford MJ. Validation of clinical classification schemes for predicting stroke: results from the National Registry of Atrial Fibrillation. JAMA. 2001;285(22):2864-70.

35. Austin PC. A comparison of 12 algorithms for matching on the propensity score. Stat Med. 2014;33(6):1057-69. doi:10.1002/ sim.6004.

36. Song X, Gandhi P, Gilligan AM, Wang C, Henriques C, Sander $\mathrm{S}$, et al. Comparison of All-Cause, stroke, and bleed-specific healthcare resource utilization among patients with non-valvular atrial fibrillation (NVAF) and newly treated with dabigatran or warfarin. Expert Rev Pharmacoecon Outcomes Res. 2017. doi:10. 1080/14737167.2013.1347041 (Epub 3 July 2017).

37. Canestaro WJ, et al. Cost-effectiveness of oral anticoagulants for treatment of atrial fibrillation. Circ Cardiovasc Qual Outcomes. 2013;6:724-31. 